Supporting Information for:

\title{
Facile Photo-crosslinking System for Polymeric Gate Dielectric Materials toward Solution-processed Organic Field-effect Transistors: Role of Crosslinker in Various Polymer Types
}

Hyeok-jin Kwon, ${ }^{\dagger, 1}$ Xiaowu Tang, ${ }^{\ddagger 1}$ Seongjun Shin, ${ }^{\S, 1}$ Jisu Hong, ${ }^{\dagger}$ Wonkyo Jeong, ${ }^{\S}$ Yohan Jo, ${ }^{\S}$ Tae Kyu An, ${ }^{\S, \#, *}$ Jihoon Lee, ${ }^{\S, \#, *}$ Se Hyun Kim, ${ }^{\ddagger}, \$, *$

${ }^{\dagger}$ Department of Chemical Engineering, Pohang University of Science and Technology, Pohang 37673, Republic of Korea

‡Department of Advanced Organic Materials Engineering, Yeungnam University, Gyeongsan, 38541, Republic of Korea

$\S$ Department of IT Convergence, Korea National University of Transportation, Chungju, 27469, Republic of Korea

\# Department of Polymer Science and Engineering, Korea National University of Transportation, Chungju 27469, Republic of Korea

\$School of Chemical Engineering, Yeungnam University, Gyeongsan 38541, Republic of Korea

${ }^{1}$ Hyeok-jin Kwon, Xiaowu Tang, and Seongjun Shin contributed equally to this study.

* Corresponding author: taekyu1985@ut.ac.kr (T.K.A.), jihoonli@ut.ac.kr (J.L), shkim97@yu.ac.kr (S.H.K)

Keywords: gate dielectrics, organic field-effect transistors, cyclic olefin copolymer, poly(vinylidene fluoride-co-hexafluoropropylene), photo-crosslinker 


\section{Experiments: Synthesis of FPA-xF $(x=3,4)$}

\section{Materials}

Pentafluorobenzoyl chloride (Acros), ethylene glycol (Acros), ethyl-2(hydroxymethyl)-1,3-propanediol (Alfa Aesar), (2,2-bis(hydroxymethyl)propane-1,3-diol (Alfa Aesar), and sodium azide (Sigma-Aldrich) were used as received without additional purification.

The known crosslinking agent ethane-1,2-diyl bis(4-azido-2,3,5,6tetrafluorobenzoate) (FPA-2F) was synthesized according to the previously reported procedure). ${ }^{1}$

\section{Measurements}

${ }^{1} \mathrm{H}-\mathrm{NMR}$ spectra were recorded using a BRUKER AVANCE $400 \mathrm{MHz}$ NMR spectrometer. ${ }^{13} \mathrm{C}$ - and ${ }^{19} \mathrm{~F}-\mathrm{NMR}$ spectra were recorded using a BRUKER AVANCE $500 \mathrm{MHz}$ NMR spectrometer. UV absorption spectra were acquired using a Hewlett Packard 8453 UVVis spectrometer. FT-IR spectra were recorded using a JASCO FT/IR-4600 FT-IR spectrometer.

\section{Synthesis of 2-ethyl-2-(((perfluorobenzoyl)oxy)methyl)propane-1,3-diyl bis(2,3,4,5,6- pentafluorobenzoate) (1)}

A mixture of 2-ethyl-2-(hydroxymethyl)propane-1,3-diol (0.8 g, $5.9 \mathrm{mmol})$ and triethylamine $(2.20 \mathrm{~g}, 21.7 \mathrm{mmol})$ in anhydrous 1,4-dioxane $(49.3 \mathrm{ml})$ was stirred at $0{ }^{\circ} \mathrm{C}$ and then pentafluorobenzoyl chloride (5 g, $21.7 \mathrm{mmol}$ ) in anhydrous 1,4-dioxane (49.3 $\mathrm{mL}$ ) was added. A white precipitate of triethylammonium chloride was observed immediately. The mixture was stirred overnight and filtered. The filtrate was diluted with chloroform and poured into an excess of $\mathrm{H}_{2} \mathrm{O}$. After separation of the organic layer, the aqueous layer was extracted with chloroform twice. The organic layers were again washed with $\mathrm{H}_{2} \mathrm{O}$, dried over anhydrous $\mathrm{MgSO}_{4}$, and then evaporated to yield a product as a white solid (3.7 g, yield 88\%). ${ }^{1} \mathrm{H}-\mathrm{NMR}$ $\left(400 \mathrm{MHz}, \mathrm{CDCl}_{3}\right) \delta(\mathrm{ppm}): 4.42(\mathrm{~s}, 2 \mathrm{H}), 1.68(\mathrm{dd}, \mathrm{J}=15.2,7.6 \mathrm{~Hz}, 1 \mathrm{H}), 1.00$ (t, J = 7.6 Hz, $1 \mathrm{H})$. 


\section{Synthesis of 2-(((4-azido-2,3,5,6-tetrafluorobenzoyl)oxy)methyl)-2-ethylpropane-1,3-diyl bis(4-azido-2,3,5,6-tetrafluorobenzoate) (FPA-3F)}

To a solution of sodium azide (2 g, $31 \mathrm{mmol})$ in water $(23.2 \mathrm{~mL})$ and acetone (23.2 $\mathrm{mL}$ ) in a $250 \mathrm{~mL}$ two-necked round-bottom flask equipped with a stirring bar and reflux condenser was added a solution of $\mathbf{1}(3.7 \mathrm{~g}, 5.2 \mathrm{mmol})$ in acetone (33.2 mL), and the mixture was stirred at $60{ }^{\circ} \mathrm{C}$ overnight. After the mixture was cooled to room temperature, the acetone was removed in vacuo. The reaction mixture was diluted with chloroform and poured into an excess of $\mathrm{H}_{2} \mathrm{O}$. After separation of the organic layer, the aqueous layer was extracted with chloroform twice. Organic layers were again washed with $\mathrm{H}_{2} \mathrm{O}$ and dried over anhydrous $\mathrm{MgSO}_{4}$. The chloroform was removed, and the crude product was purified using flash column chromatography on silica with chloroform as the eluant. The resulting solid was recrystallized from chloroform $/ n$-hexane to obtain the desired compound as a white solid (2.4 g, yield 59\%). ${ }^{1} \mathrm{H}-\mathrm{NMR}\left(400 \mathrm{MHz}, \mathrm{CDCl}_{3}\right) \delta(\mathrm{ppm}): 4.41$ (s, 6H), 1.67 (q, J = 7.6 Hz, 2H), 0.99 (t, J = 7.6 $\mathrm{Hz}, 3 \mathrm{H}) .{ }^{13} \mathrm{C}-\mathrm{NMR}\left(125 \mathrm{MHz}, \mathrm{CDCl}_{3}\right) \delta(\mathrm{ppm}): 159.05$ (s), 146.81 (d, J = $\left.12.6 \mathrm{~Hz}\right), 144.95-$ 144.62 (m), 141.68 (d, J = 18.3 Hz), 139.68 (d, J = 17.6 Hz), 124.20 (t, J = 11.9 Hz), 106.93 (t, $\mathrm{J}=14.7 \mathrm{~Hz}$ ), 65.62 (s), 41.15 (s), 22.92 (s), 7.37 (s). ${ }^{19} \mathrm{~F}-\mathrm{NMR}\left(470 \mathrm{MHz}, \mathrm{CDCl}_{3}, \mathrm{AA}^{\prime} \mathrm{XX}^{\prime}\right) \delta$ (ppm) : -138.18 to $-138.32(\mathrm{~m}),--150.45$ to $-150.59(\mathrm{~m})$. UV-Vis $\lambda_{\max }($ in $M C)=267 \mathrm{~nm}$, $\lambda_{\max }($ spin-coated film $)=272 \mathrm{~nm}$. FT-IR 2130, 1739, 1647, 1483, 1256, $1009 \mathrm{~cm}^{-1}$.

\section{Synthesis of 2,2-bis(((perfluorobenzoyl)oxy)methyl)propane-1,3-diyl bis(2,3,4,5,6- pentafluorobenzoate) (2)}

A mixture of 2,2-bis(hydroxymethyl)propane-1,3-diol (0.75 g, $5.4 \mathrm{mmol}$ ) and triethylamine (3.30 g, $32.6 \mathrm{mmol})$ in anhydrous 1,4 -dioxane $(70.5 \mathrm{~mL})$ was stirred at $0{ }^{\circ} \mathrm{C}$, and then a solution of pentafluorobenzoyl chloride (7.5 g, $32.6 \mathrm{mmol})$ in anhydrous 1,4-dioxane (70.5 mL) was added. A white precipitate of triethylammonium chloride was observed immediately. The mixture was stirred overnight and filtered. The filtrate was diluted with chloroform and poured into an excess of $\mathrm{H}_{2} \mathrm{O}$. After separation of the organic layer, the aqueous layer was extracted twice with chloroform. Organic layers were again washed with $\mathrm{H}_{2} \mathrm{O}$, dried over $\mathrm{MgSO}_{4}$, and then evaporated to yield a product as a white solid (3.8 g, yield 72\%). ${ }^{1} \mathrm{H}-\mathrm{NMR}\left(400 \mathrm{MHz}, \mathrm{CDCl}_{3}\right) \delta(\mathrm{ppm}): 4.57$ (s, 1H) 
Synthesis of 2,2-bis(((4-azido-2,3,5,6-tetrafluorobenzoyl)oxy)methyl)propane-1,3-diyl bis(4azido-2,3,5,6-tetrafluorobenzoate) (FPA-4F)

To a solution of sodium azide $(2.17 \mathrm{~g}, 33.3 \mathrm{mmol})$ in water $(24.1 \mathrm{~mL})$ and acetone (24.1 mL) in a $250 \mathrm{~mL}$ two-necked round-bottom flask equipped with a stirring bar and reflux condenser was added a solution of 2 (3.8 g, $4.2 \mathrm{mmol})$ in acetone (34.5 mL), and the resultant mixture was stirred at $60{ }^{\circ} \mathrm{C}$ overnight. After the mixture was cooled to room temperature, the acetone was removed in vacuo. The reaction mixture was diluted with chloroform and poured into an excess of H2O. After separation of the organic layer, the aqueous layer was extracted with chloroform twice. Organic layers were again washed with $\mathrm{H}_{2} \mathrm{O}$ and dried over anhydrous $\mathrm{MgSO}_{4}$. The chloroform was removed, and the crude product was purified using flash column chromatography on silica with chloroform as the eluant. The resulting solid was recrystallized from chloroform $/ n$-hexane to obtain the desired compound as a white solid (2.6 g, yield 60\%). ${ }^{1} \mathrm{H}-\mathrm{NMR}\left(400 \mathrm{MHz}, \mathrm{CDCl}_{3}\right) \delta(\mathrm{ppm}): 4.55$ (s, 1H). ${ }^{13} \mathrm{C}-\mathrm{NMR}(125 \mathrm{MHz}, \mathrm{CDCl} 3) \delta(\mathrm{ppm})$ : 158.74 (s), 147.06-146.79 (m), 144.89 (dd, J = 11.8, 6.5 Hz), 141.70 (d, J = 14.1 Hz), 139.89139.42 (m), 124.61 (t, J = 11.8 Hz), 106.35 (t, J = 14.3 Hz), 63.77 (s), 42.42 (s). ${ }^{19} \mathrm{~F}$ NMR (470 $\left.\mathrm{MHz}, \mathrm{CDCl}_{3}\right) \delta(\mathrm{ppm}):-137.91$ to $-138.08(\mathrm{~m}),-150.19$ to $-150.34(\mathrm{~m})$. UV-Vis $\lambda_{\max }$ (in $\mathrm{MC})=269 \mathrm{~nm}, \lambda_{\max }($ spin-coated film $)=275 \mathrm{~nm}$. FT-IR 2137, 1741, 1648, 1491, 1330, 1258, 1203, $1007 \mathrm{~cm}^{-1}$. 


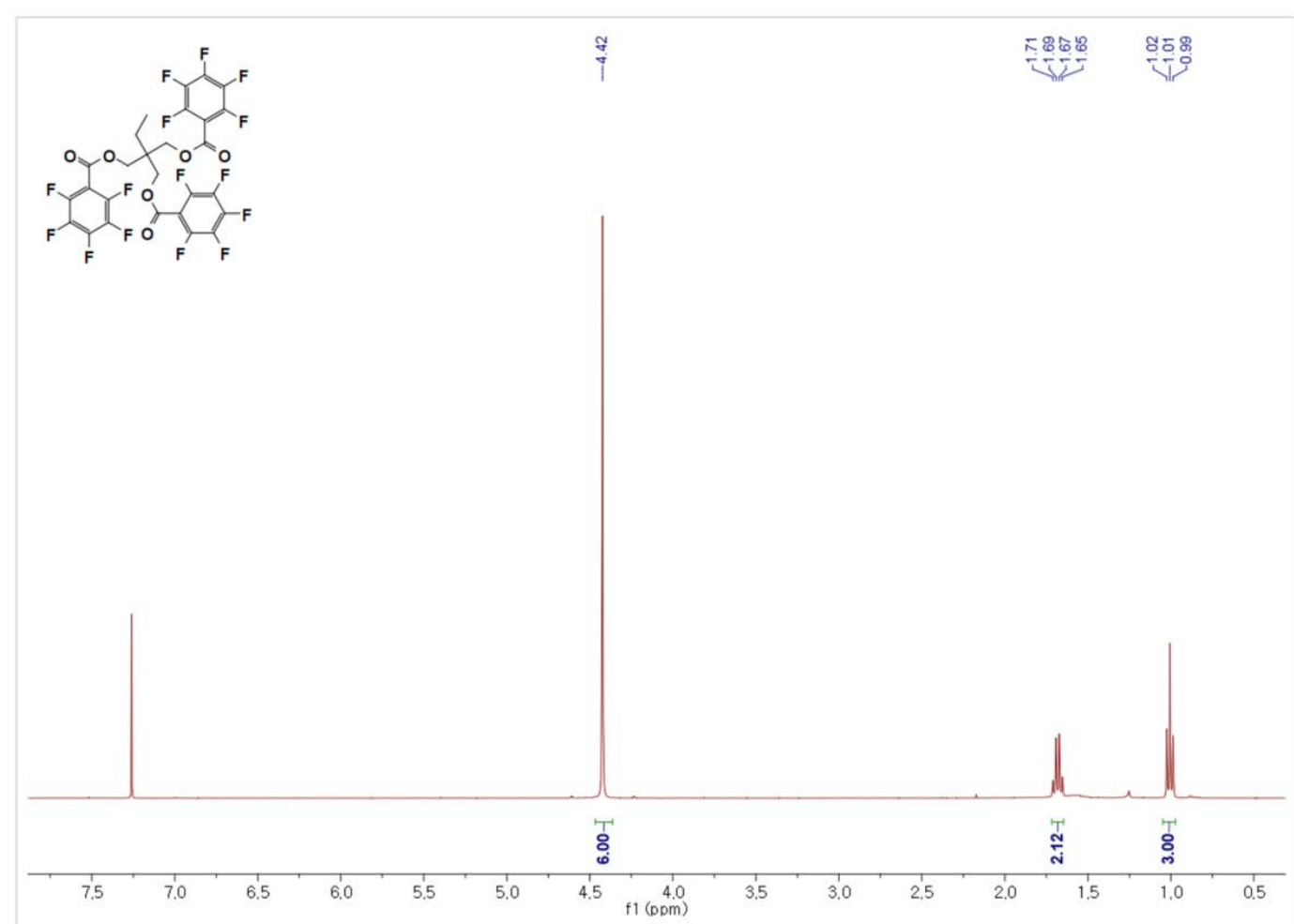

Figure S1. ${ }^{1} \mathrm{H}-\mathrm{NMR}$ spectrum of 2-ethyl-2-(((perfluorobenzoyl)oxy)methyl)propane-1,3-diyl bis(2,3,4,5,6-pentafluorobenzoate) (in $\left.\mathrm{CDCl}_{3}\right)$.

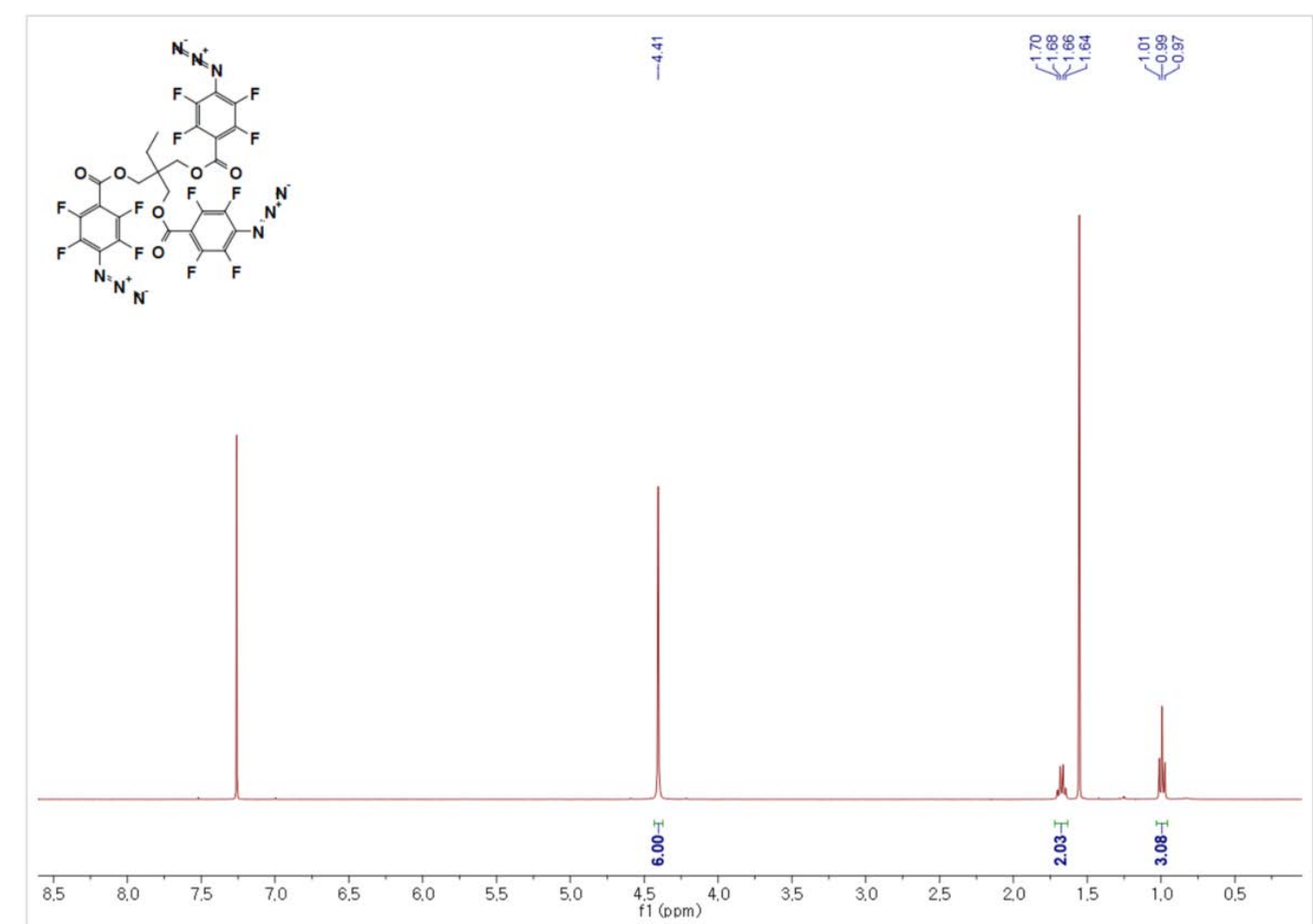

Figure S2. ${ }^{1} \mathrm{H}-\mathrm{NMR}$ spectrum of 2-((4-azido-2,3,5,6-tetrafluorobenzoyl)oxy)methyl)-2ethylpropane-1,3-diyl bis(4-azido-2,3,5,6-tetrafluorobenzoate) (in $\mathrm{CDCl}_{3}$ ). 


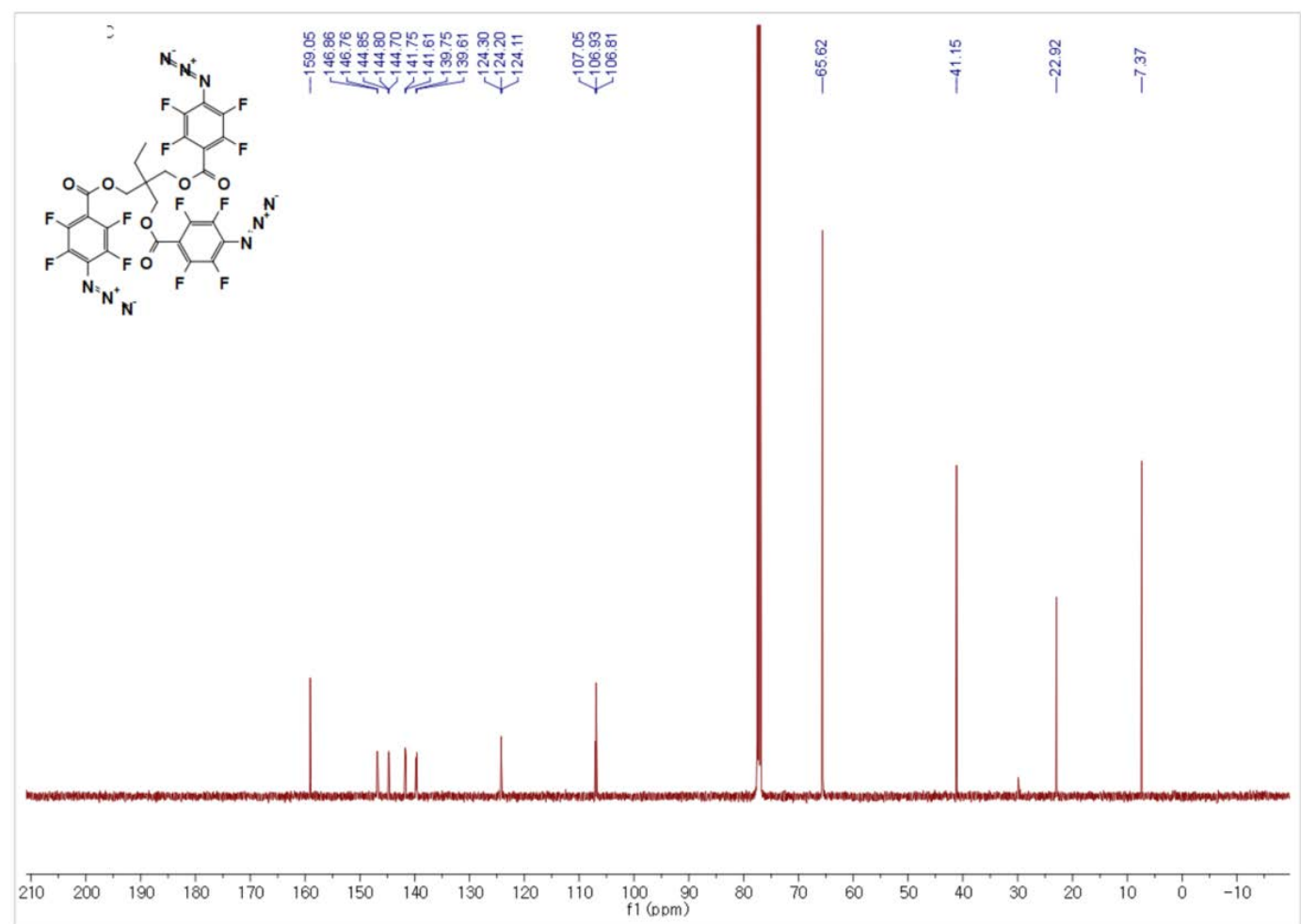

Figure S3. ${ }^{13} \mathrm{C}-\mathrm{NMR}$ spectrum of 2-(((4-azido-2,3,5,6-tetrafluorobenzoyl)oxy)methyl)-2ethylpropane-1,3-diyl bis(4-azido-2,3,5,6-tetrafluorobenzoate) (in $\mathrm{CDCl}_{3}$ ).

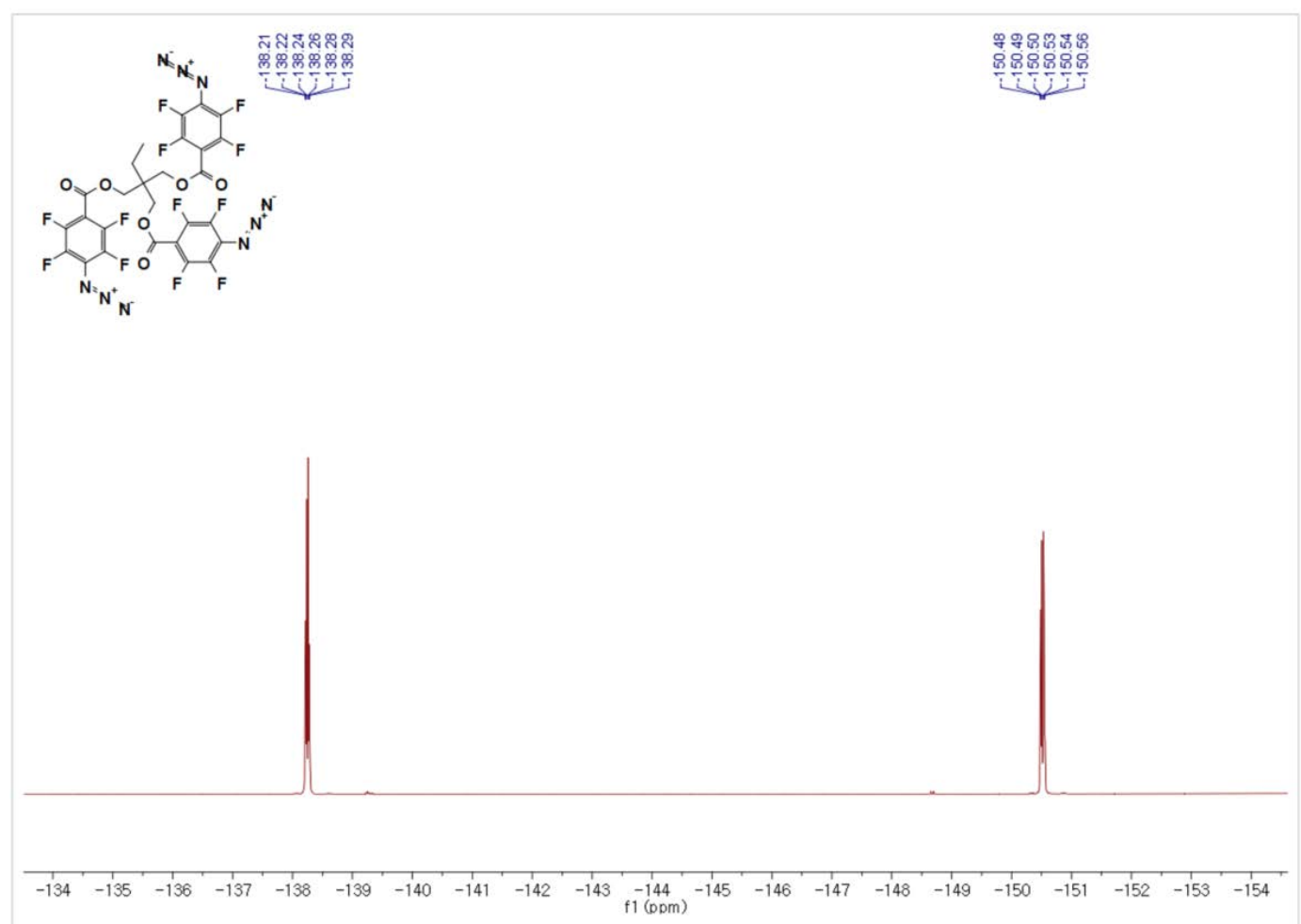

Figure S4. ${ }^{19}$ F-NMR spectrum of 2-(((4-azido-2,3,5,6-tetrafluorobenzoyl)oxy)methyl)-2ethylpropane-1,3-diyl bis(4-azido-2,3,5,6-tetrafluorobenzoate) (in $\mathrm{CDCl}_{3}$ ). 


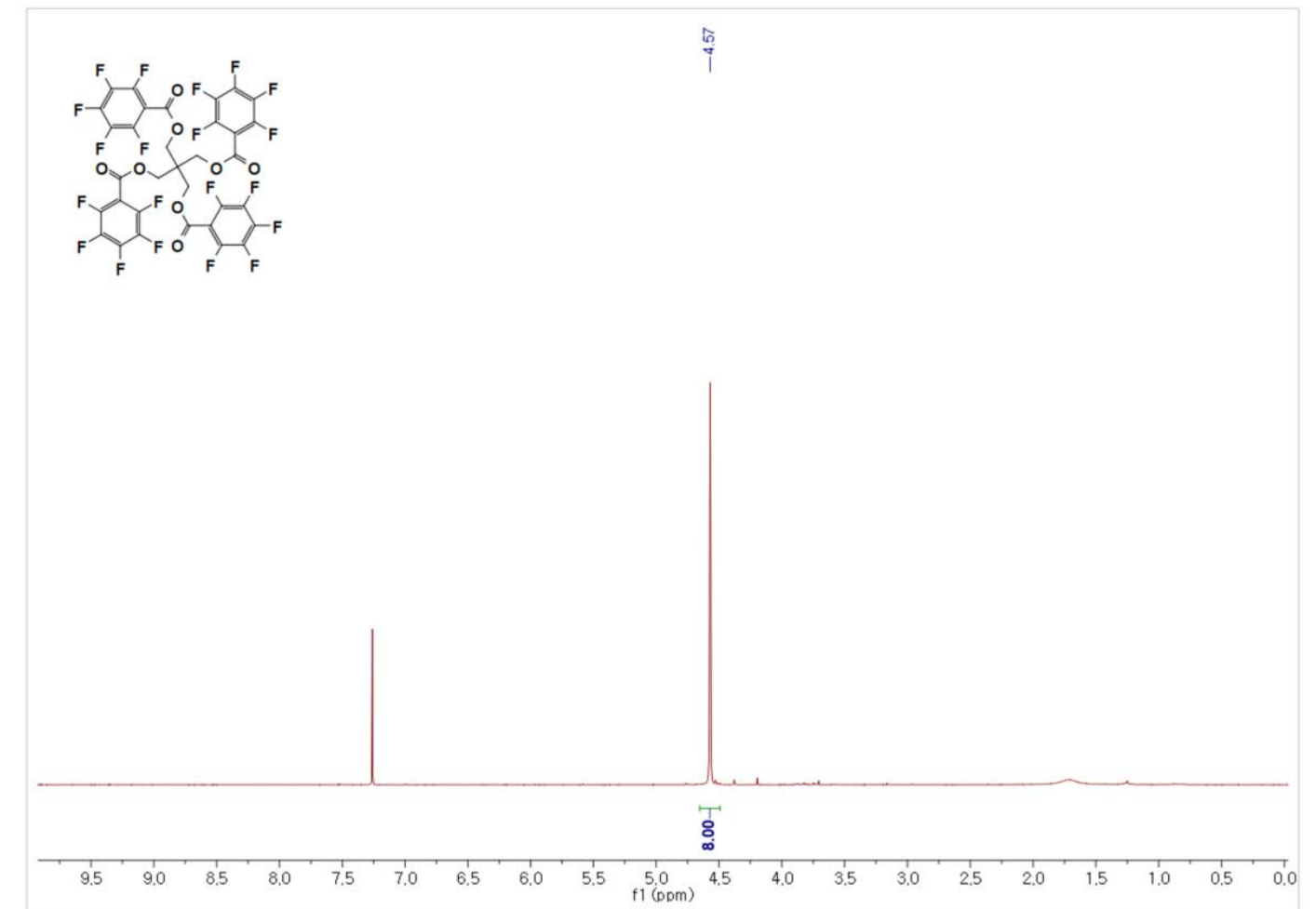

Figure S5. ${ }^{1} \mathrm{H}-\mathrm{NMR}$ spectrum of 2,2-bis(((perfluorobenzoyl)oxy)methyl)propane-1,3-diyl bis(2,3,4,5,6-pentafluorobenzoate) (in $\left.\mathrm{CDCl}_{3}\right)$.

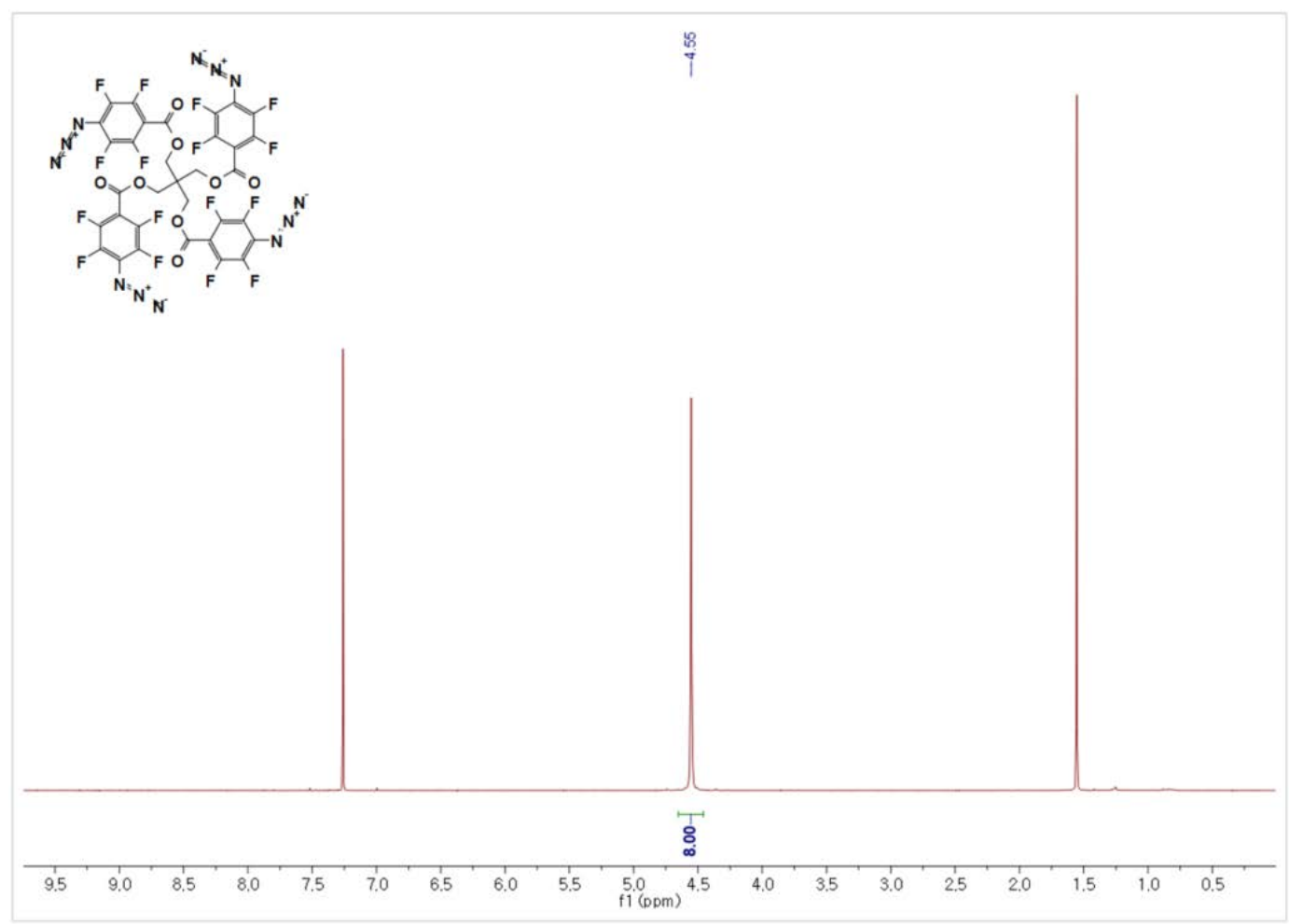

Figure S6. $\quad{ }^{1} \mathrm{H}-\mathrm{NMR}$ spectrum of 2,2-bis(((4-azido-2,3,5,6-tetrafluorobenzoyl) oxy)methyl)propane-1,3-diyl bis(4-azido-2,3,5,6-tetrafluorobenzoate) (in $\mathrm{CDCl}_{3}$ ). 


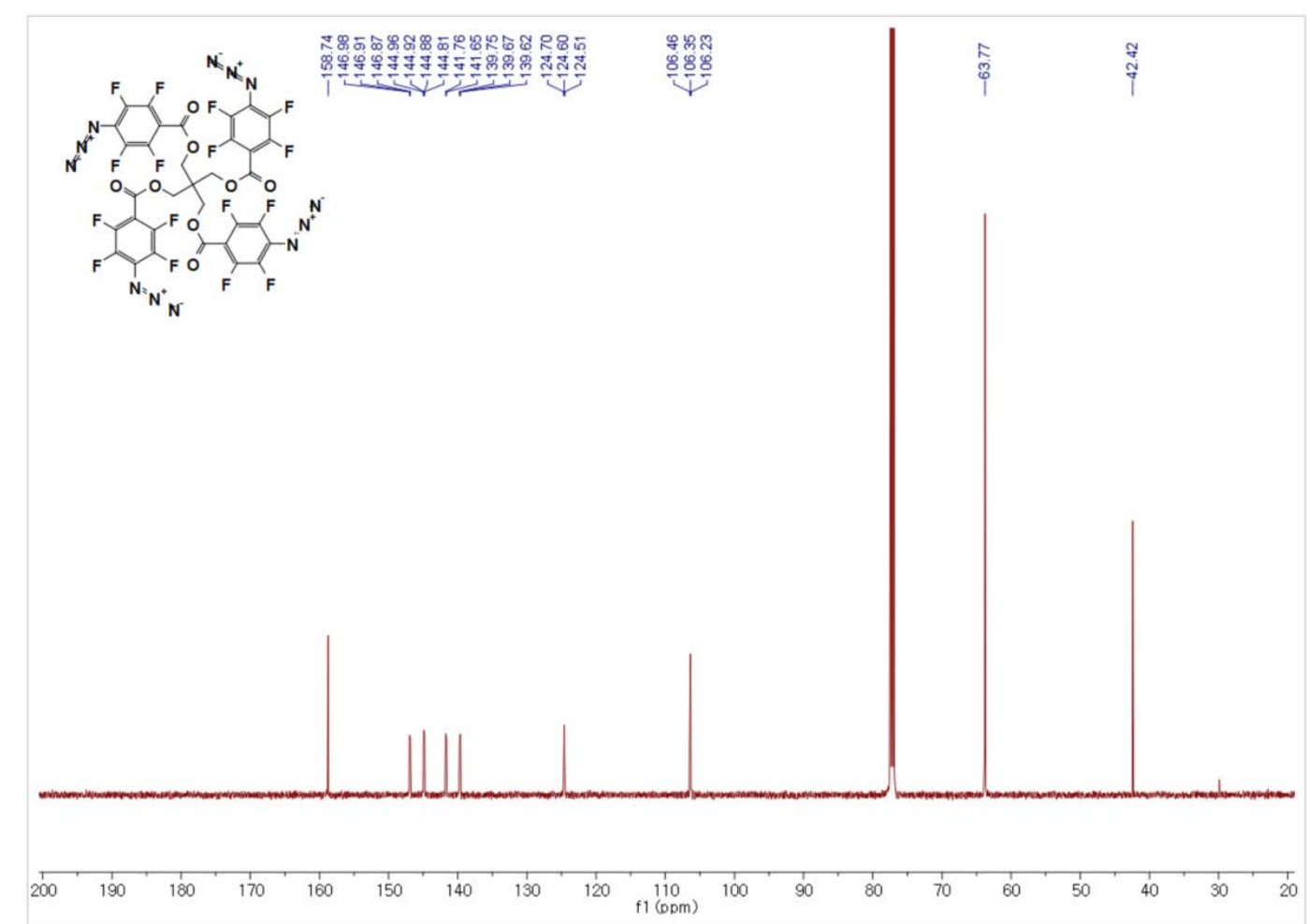

Figure S7. ${ }^{13} \mathrm{C}-\mathrm{NMR}$ spectrum of 2,2-bis(((4-azido-2,3,5,6-tetrafluorobenzoyl) oxy)methyl)propane-1,3-diyl bis(4-azido-2,3,5,6-tetrafluorobenzoate) (in $\mathrm{CDCl}_{3}$ ).

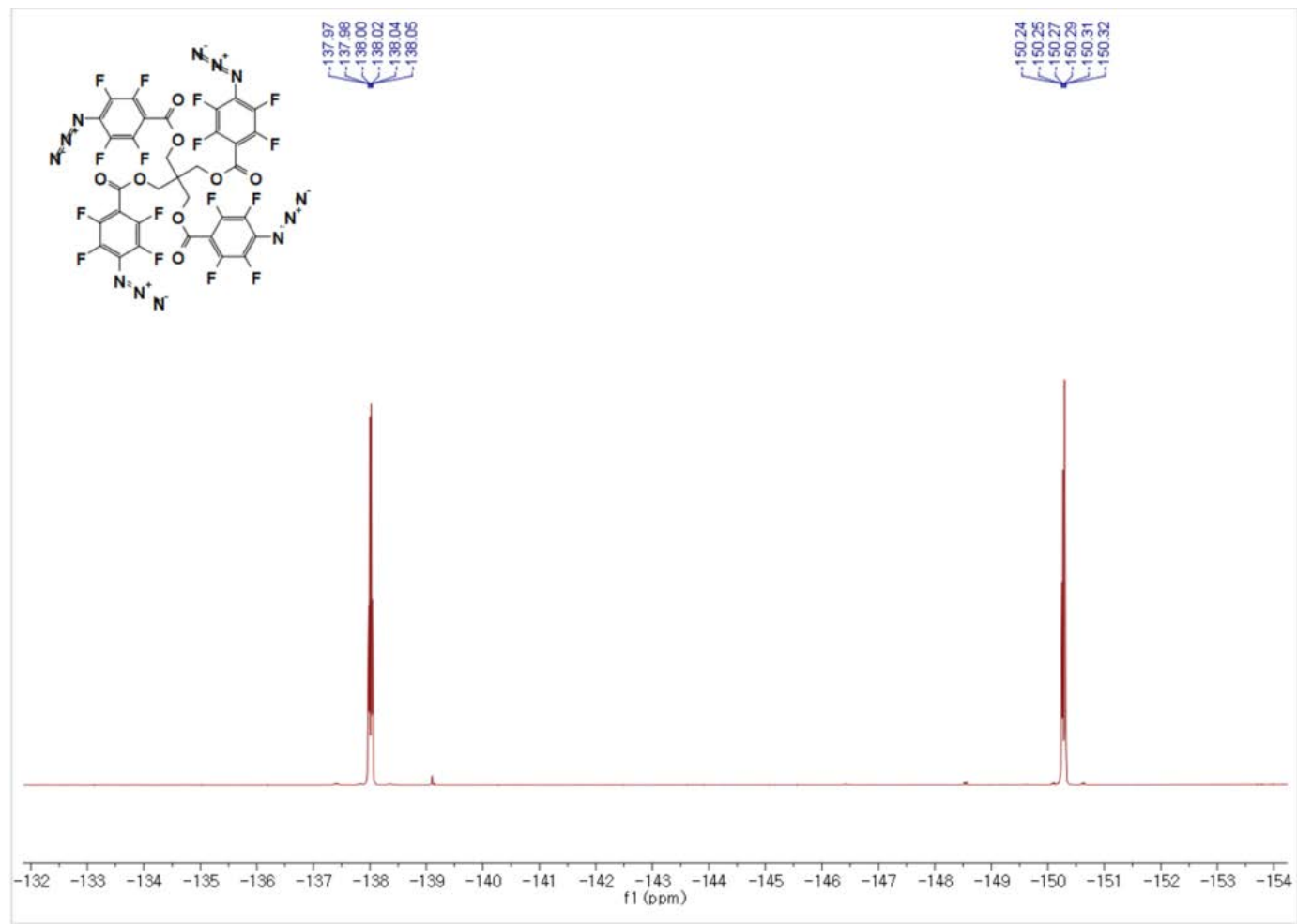

Figure S8. ${ }^{19}$ F-NMR spectrum of 2,2-bis(((4-azido-2,3,5,6-tetrafluorobenzoyl) oxy)methyl)propane-1,3-diyl bis(4-azido-2,3,5,6-tetrafluorobenzoate) (in $\mathrm{CDCl}_{3}$ ). 


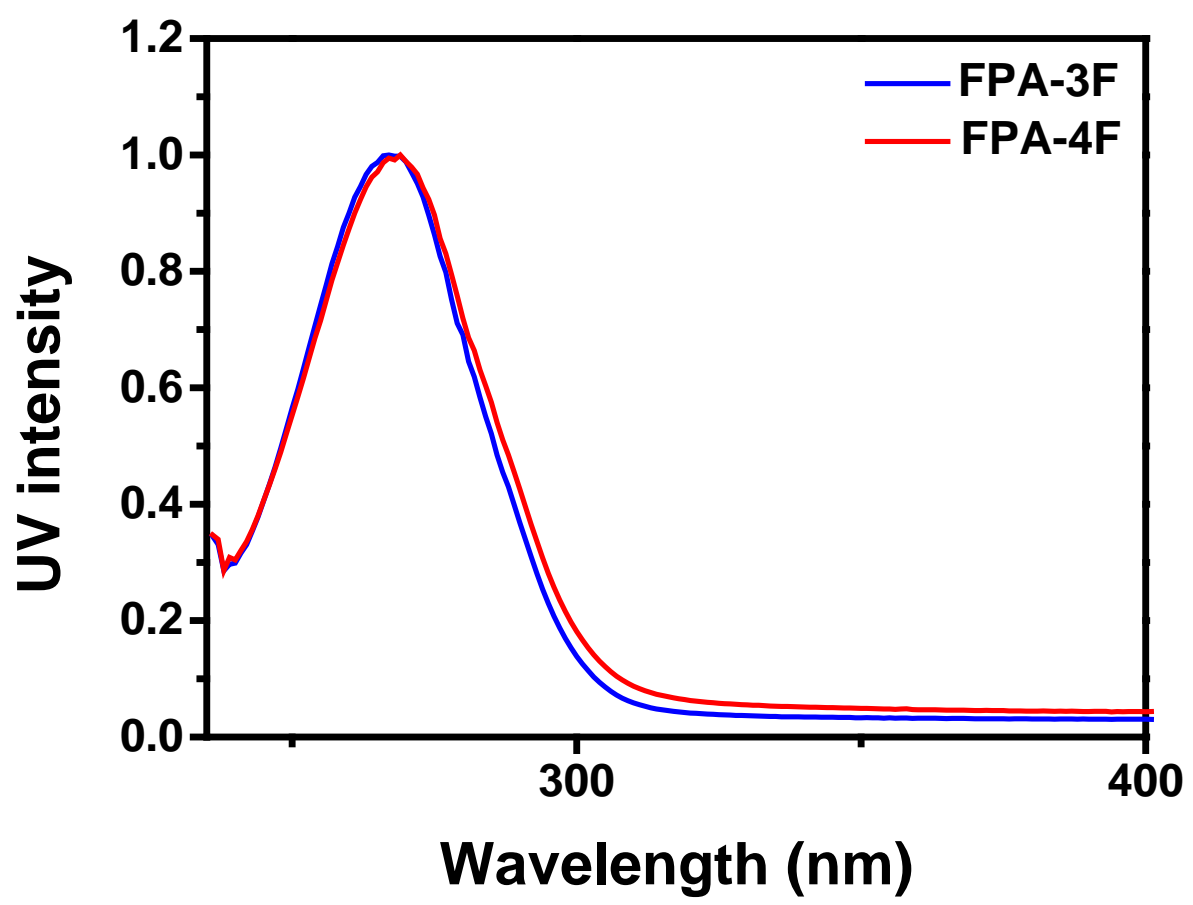

Figure S9 UV-Vis spectrum of FPA- $x F$ series (in MC).

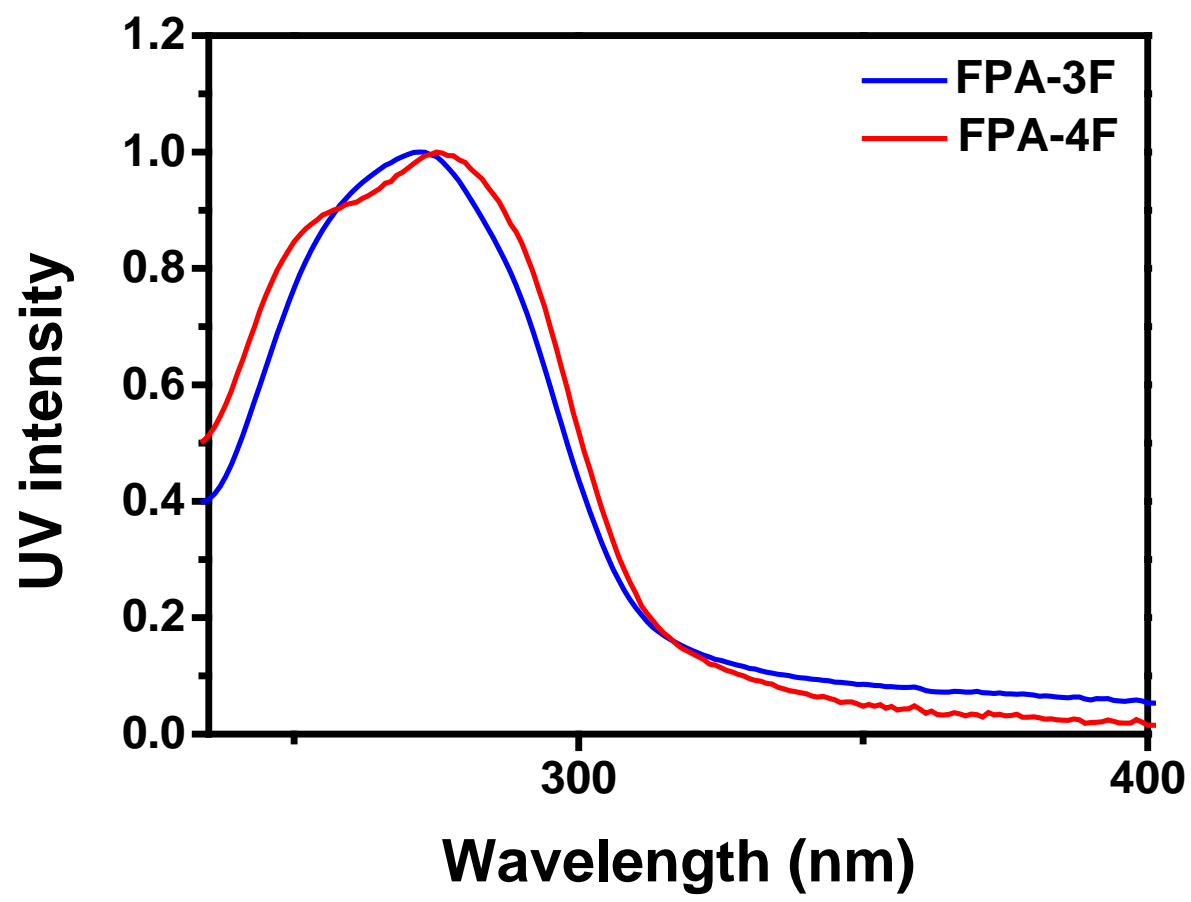

Figure S10. UV-Vis spectrum of the FPA-xF series (film). 


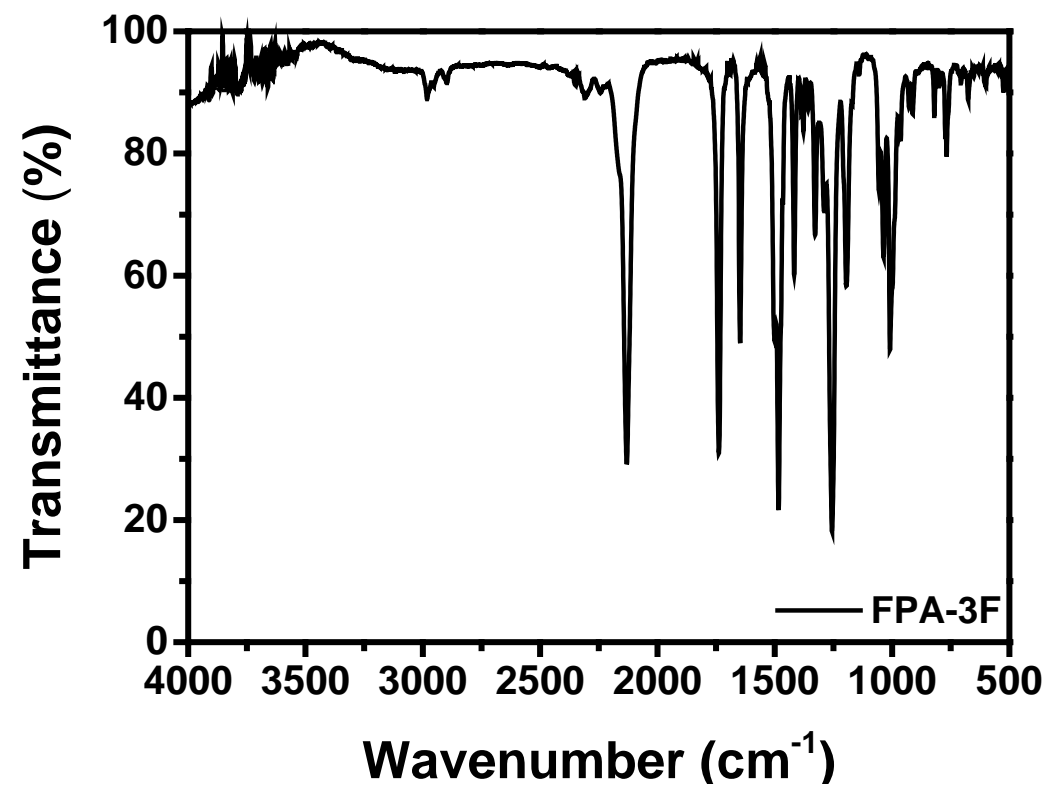

Figure S11. FT-IR spectrum of 2-(((4-azido-2,3,5,6-tetrafluorobenzoyl)oxy)methyl)-2ethylpropane-1,3-diyl bis(4-azido-2,3,5,6-tetrafluorobenzoate).

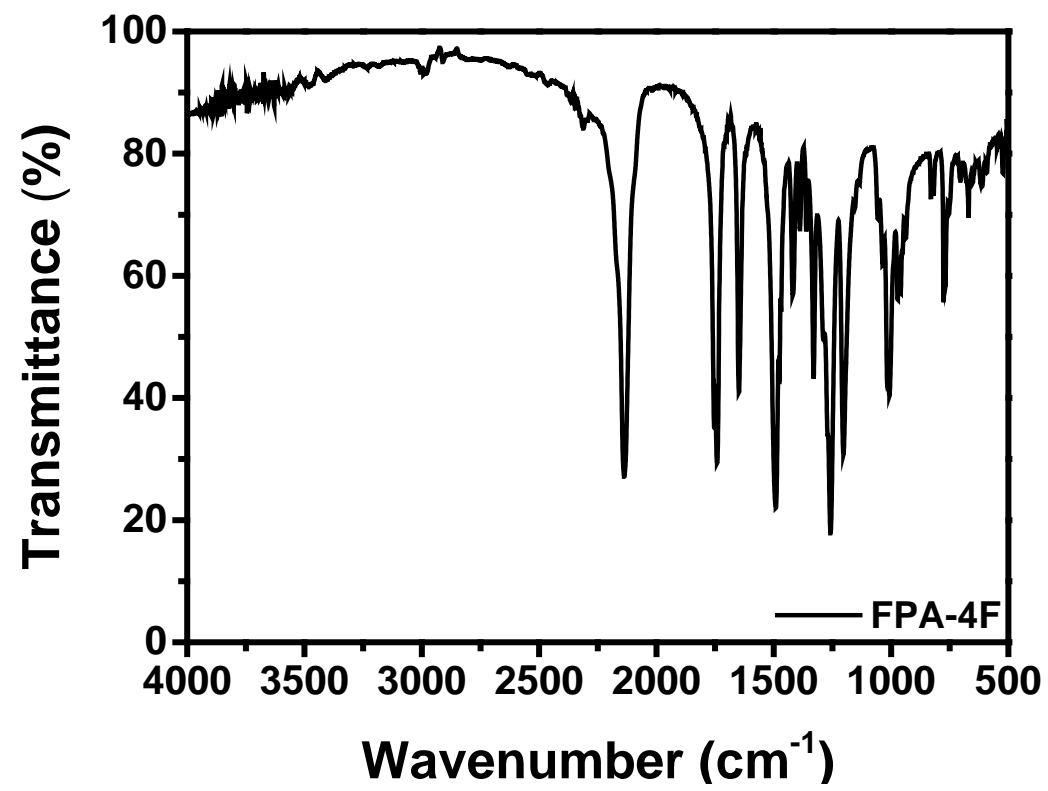

Figure S12. FT-IR spectrum of 2,2-bis(((4-azido-2,3,5,6-tetrafluorobenzoyl) oxy)methyl)propane-1,3-diyl bis(4-azido-2,3,5,6-tetrafluorobenzoate). 
(a)
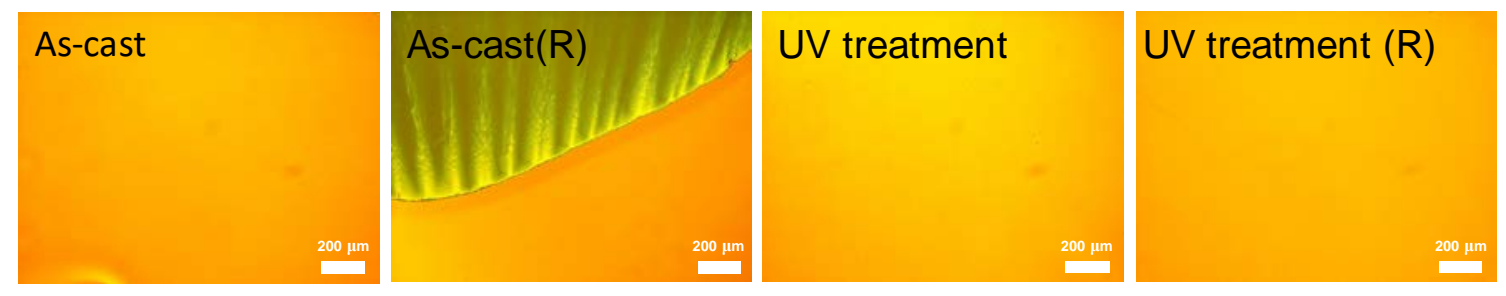

(b)
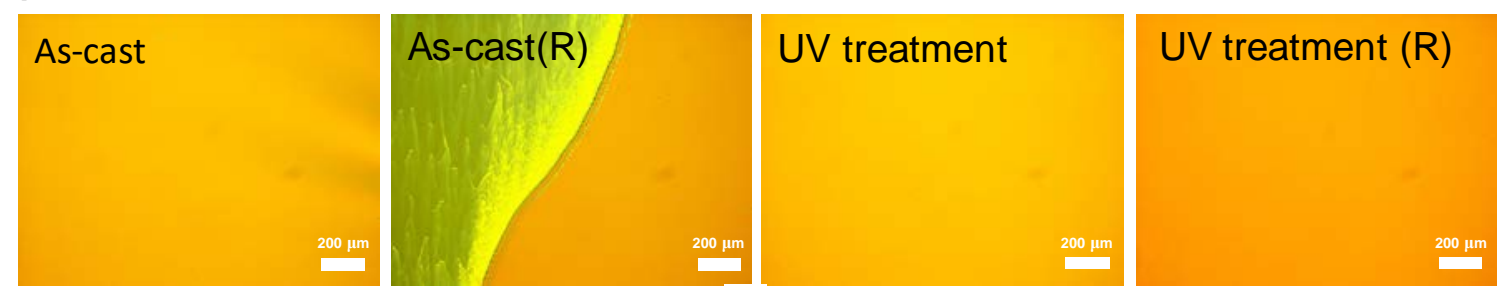

(c)
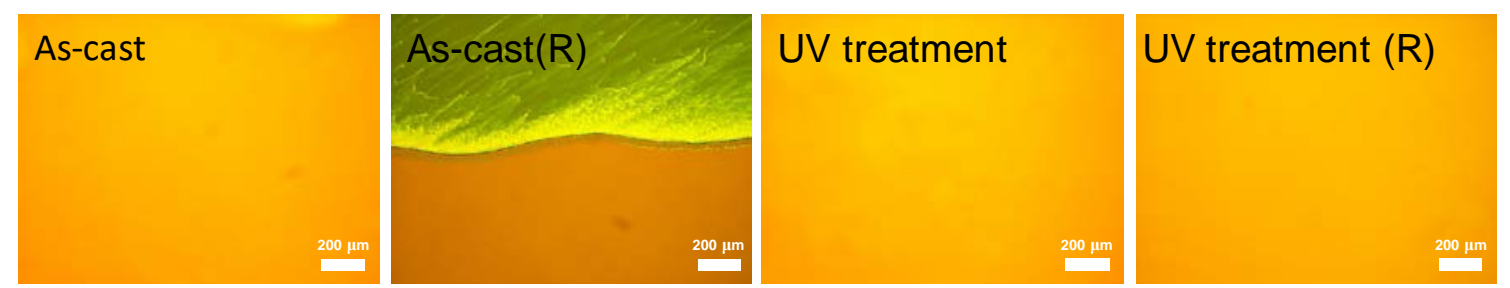

Figure S13. OM image of deposited FCOC series films: (a) 2FCOC, (b) 3FCOC, and (c) 4FCOC.
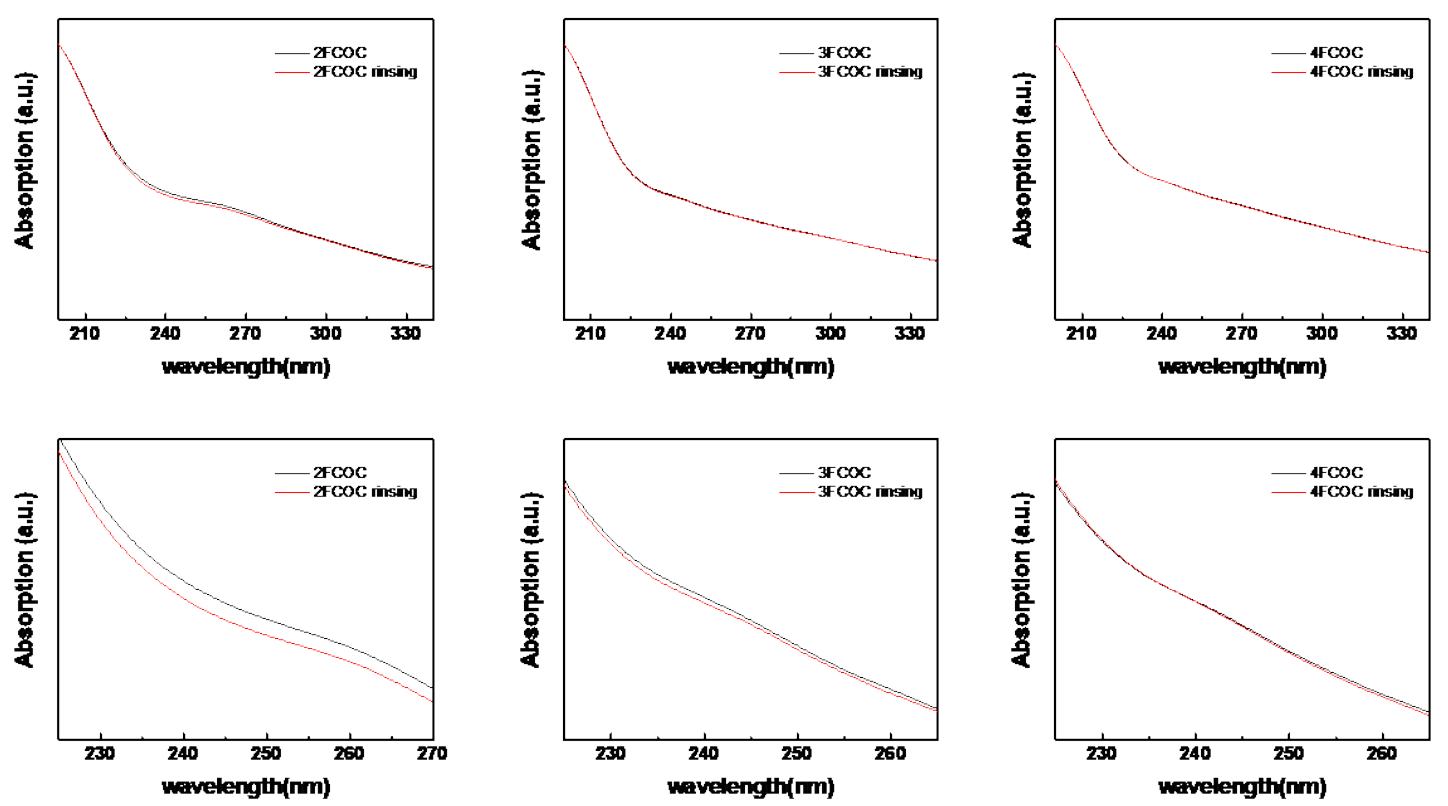

Figure S14. UV-Vis absorption spectra of FCOC series films with toluene rinsing process: (a) wide-range data (200-340 $\mathrm{nm}$ ) and (b) narrow-range data narrow-range data with same $\mathrm{x}$ and y axis range values.. 
(a)
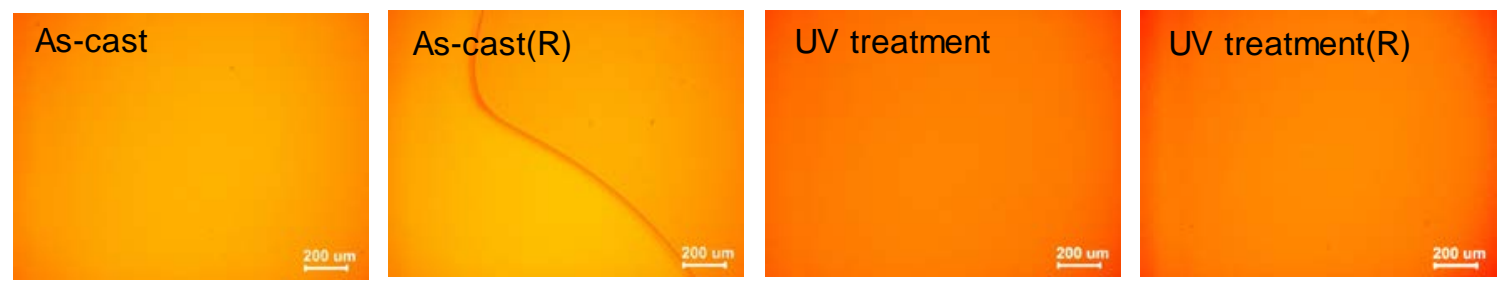

(b)
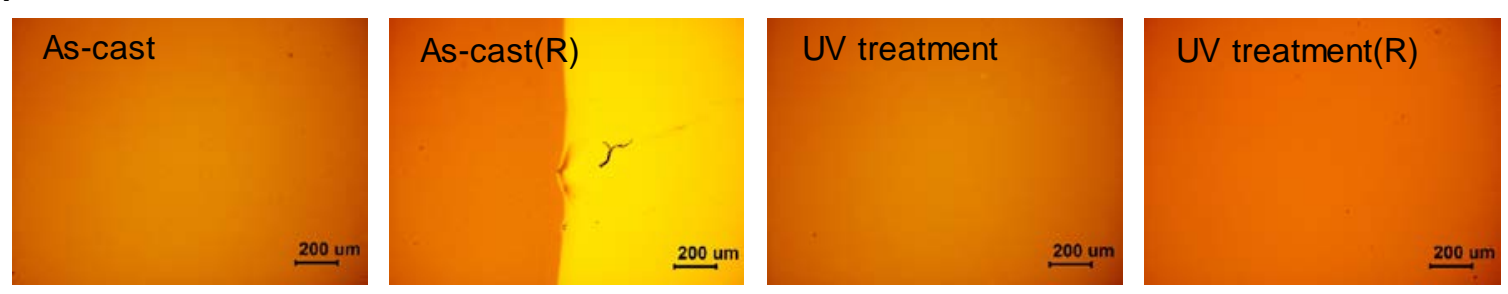

(c)
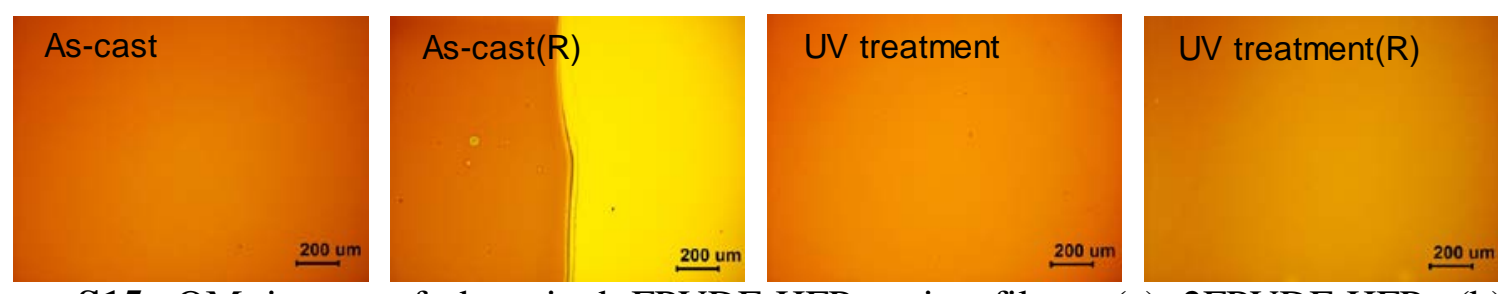

Figure S15. OM image of deposited FPVDF-HFP series films: (a) 2FPVDF-HFP, (b) 3FPVDF-HFP, and (c) 4FPVDF-HFP.

\section{Supporting note \#1.}

As described in Figure S13 and Figure S15, the dielectric layer of the FCOC and FPVDFHFP series films were successfully coated onto substrates, resulting in smooth coating morphology in macroscales. Naturally, the $x$ FCOC and $x$ FPVDF-HFP films $(x=2,3$ and 4) without crosslinking reaction showed weak solvent resistance by delamination phenomenon when rinsed with toluene and acetone, respecitively. However, when irradiated with deep-UV ( $\sim 254 \mathrm{~nm}$ ), the crosslinked films withstood rinsing with toluene and acetone, as shown in the fourth figure of all sample cases. Moreover, the UV-vis absorption spectrum of these samples before and after rinsing more clearly shows the degree of crosslinking reaction, indicating the chemical robustness. As can be seen in Figure S14a, all crosslinked FCOC film series are similar in overall shape (low difference before and after rinsing process), but exhibit a very small reduction in modification. Indeed, an enlarged range of these spectra shows a tendency for the degree of reduction to decrease as the reactor of the crosslinking agent increases. 
Therefore, our designed crosslinking agent shows high crosslinking efficiency, especially in the case of FPA-4F, it shows almost complete crosslinking reaction with COC. These observations are clear evidence of a successful crosslinking reaction, indicating the chemical robustness of films.

Table S1. Surface properties of various FCOC dielectric layers

\begin{tabular}{|c|c|c|c|c|c|}
\hline \multirow[t]{2}{*}{ Surface } & \multicolumn{2}{|c|}{ Contact Angle [ ${ }^{\circ}$ ] } & \multirow{2}{*}{$\begin{array}{c}\gamma_{s}^{\rho} \\
{\left[\mathrm{mJ} \mathrm{m}^{-2}\right]}\end{array}$} & \multirow{2}{*}{$\begin{array}{c}\gamma_{s}^{d} \\
{\left[\mathrm{~mJ} \mathrm{~m}^{-2}\right]}\end{array}$} & \multirow{2}{*}{$\begin{array}{c}\gamma_{s} \\
{\left[\mathrm{~mJ} \mathrm{~m}^{-2}\right]}\end{array}$} \\
\hline & Water & Diiodomethane & & & \\
\hline Pristine COC & 96 & 40 & 0.12 & 40.51 & 40.63 \\
\hline As-cast 2FCOC & 96 & 41 & 0.14 & 39.90 & 40.05 \\
\hline UV-treated 2FCOC & 97 & 40 & 0.06 & 40.80 & 40.86 \\
\hline As-cast 3FCOC & 97 & 40 & 0.06 & 40.80 & 40.86 \\
\hline UV-treated 3FCOC & 98 & 40 & 0.02 & 41.08 & 41.10 \\
\hline As-cast 4FCOC & 97 & 41 & 0.07 & 40.18 & 40.25 \\
\hline UV-treated 4FCOC & 97 & 40 & 0.06 & 40.80 & 40.86 \\
\hline $\begin{array}{c}\text { Prinstine } \\
\text { PVDF-HFP }\end{array}$ & 84 & 64 & 6.33 & 22.06 & 28.39 \\
\hline $\begin{array}{c}\text { As-cast } \\
\text { 2FPVDF-HFP }\end{array}$ & 83 & 64 & 6.86 & 21.85 & 28.71 \\
\hline $\begin{array}{l}\text { UV-treated } \\
\text { 2FPVDF-HFP }\end{array}$ & 84 & 66 & 6.79 & 20.82 & 27.61 \\
\hline $\begin{array}{c}\text { As-cast } \\
\text { 3FPVDF-HFP }\end{array}$ & 84 & 64 & 6.33 & 22.06 & 28.39 \\
\hline $\begin{array}{l}\text { UV-treated } \\
\text { 3FPVDF-HFP }\end{array}$ & 86 & 66 & 5.76 & 21.23 & 26.99 \\
\hline $\begin{array}{c}\text { As-cast } \\
\text { 4FPVDF-HFP }\end{array}$ & 85 & 63 & 5.61 & 22.91 & 28.52 \\
\hline $\begin{array}{l}\text { UV-treated } \\
\text { 4FPVDF-HFP }\end{array}$ & 89 & 64 & 3.99 & 23.14 & 27.13 \\
\hline
\end{tabular}




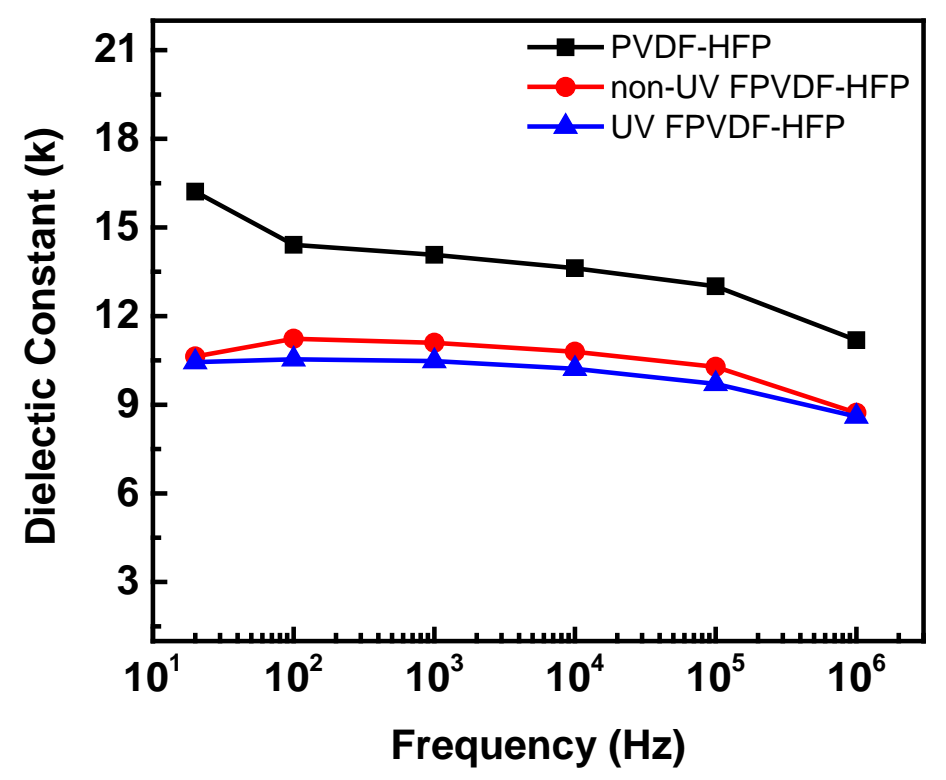

Figure S16. Dielectric constant plot of PVDF-HFP and 3FPVDF-HFP with or without UVirradiation depending on the frequency.

(a)

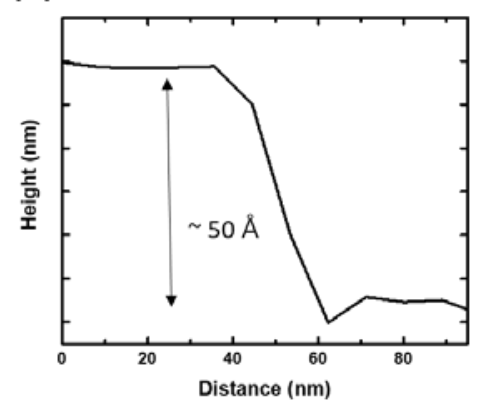

(b)

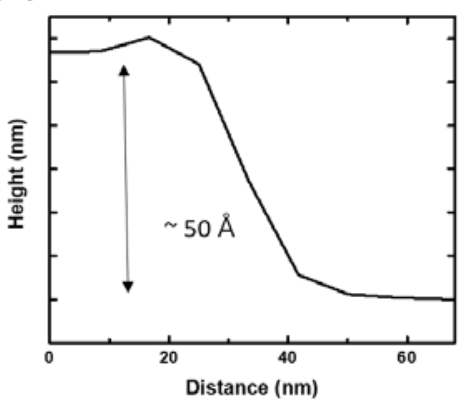

(c)

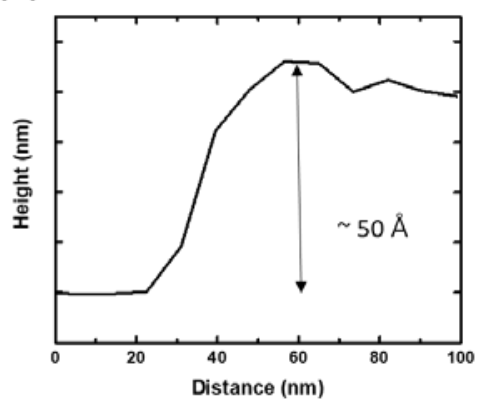

Figure S17. AFM cross-sectional height profiles PTCDI-C13 thin films on the gate dielectrics:

(a) 2FCOC, (b) 3FCOC, and (c) 4FCOC. 
(a)

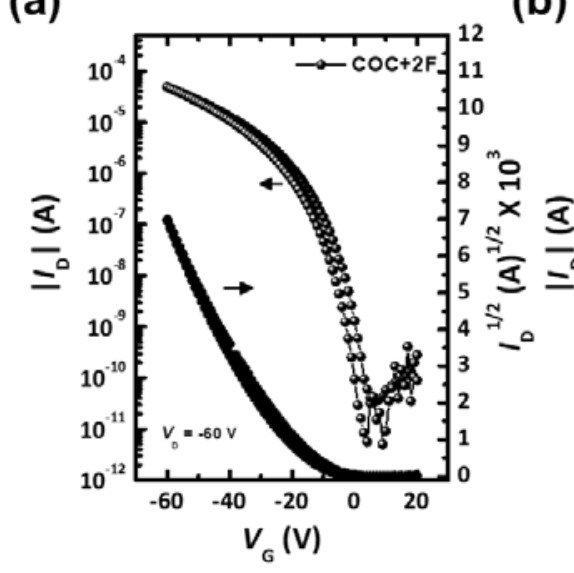

(b)

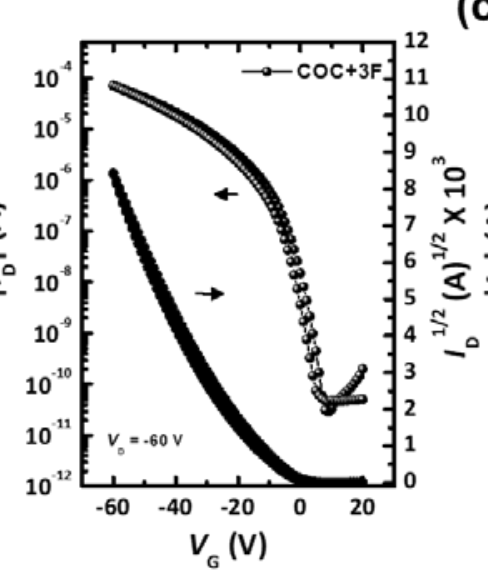

(c)

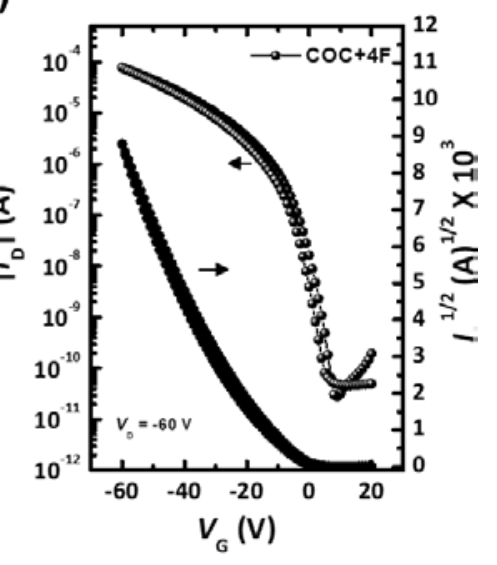

Figure S18. Transfer characteristics of $\mathrm{C}_{10}$-DNTT devices with non-UV-irradiated devices:

(a) 2FCOC, (b) 3FCOC, and (c) 4FCOC. 
(a)

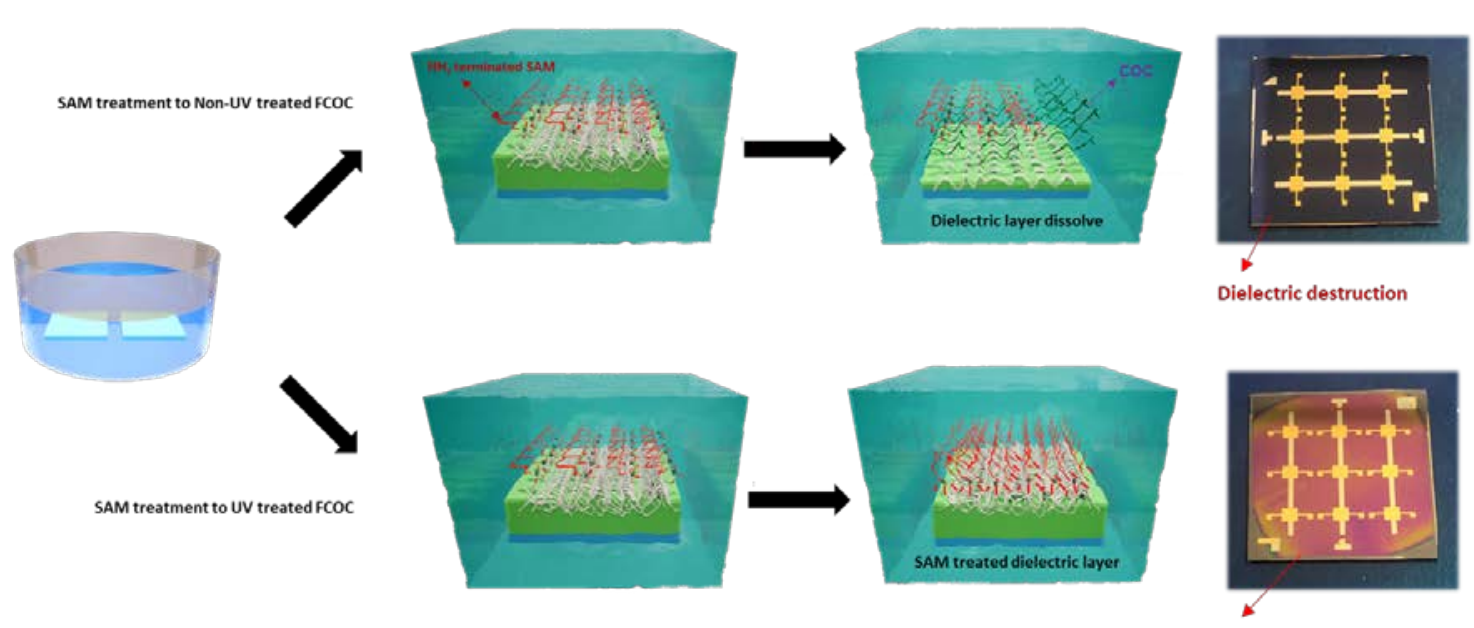

(b)

$\mathrm{NH}_{2}$ terminated dielectric surface
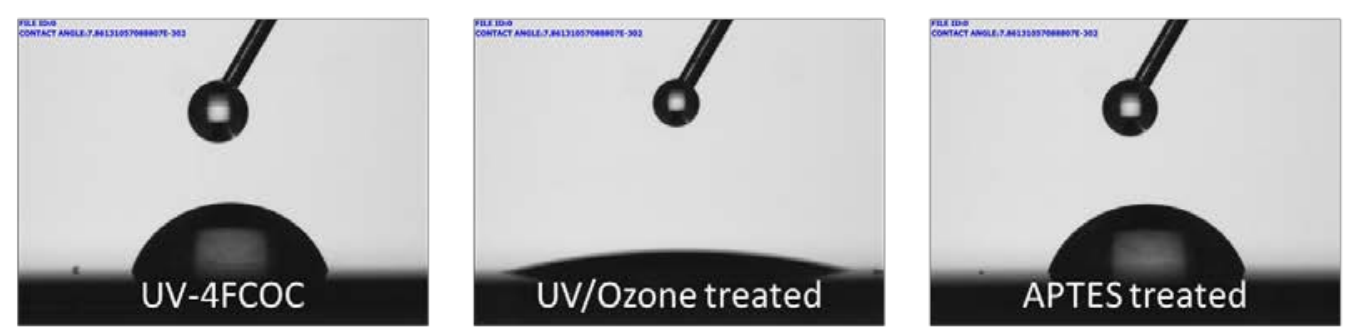

(c)
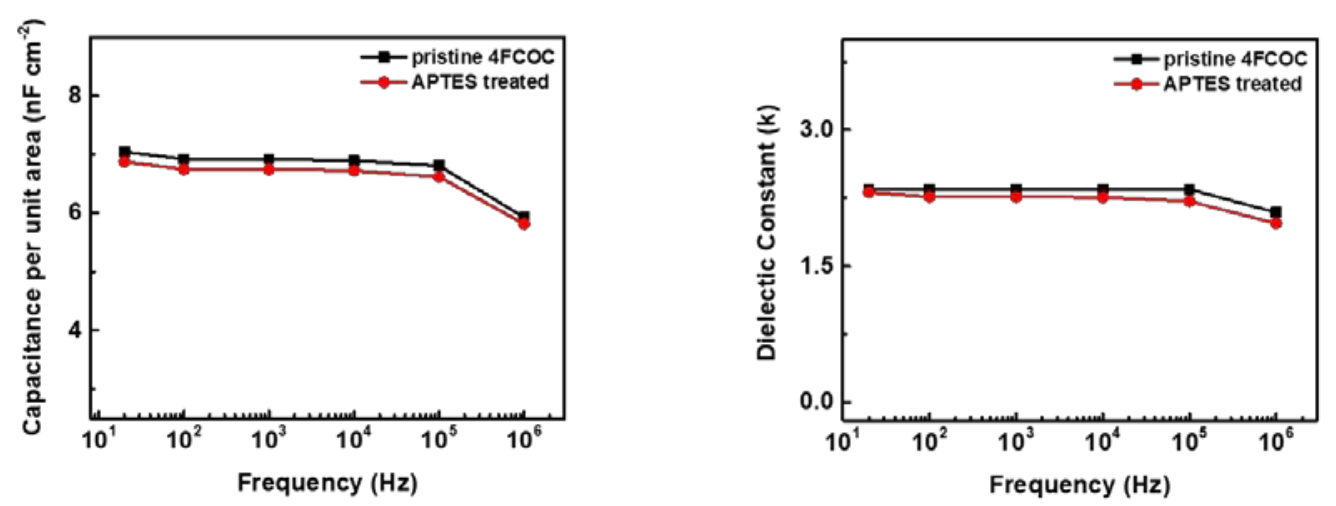

Figure S19. (a) Schematic of the APTES treatment process with or without UV irradiation to 4FCOC and real images of CNT-based devices; (b) change in contact angle of UV-treated 4FCOC after the APTES treatment process. (c) Capacitance per unit area and dielectric constant values depending on the APTES treatment. 
Supporting note \#2.

As described in Figure S18a, the APTES treatments process of dielectrics were performed by the dipping in the solution with volumic ratio 1:99 of APTES:Toulene. Since pristine COC or 4FCOC cannot form a chemical bond with APTES, UV/ozone treatment is performed before dipping to induce surface hydroxylation. ${ }^{2}$ After UV/ozoen treatement, dipping process was conducted for 1 hours. Then, the surface modified samples were rinsed and dried with toluene and Nitrogen blowing, and annealed in $100{ }^{\circ} \mathrm{C}$ under vacuum state for $30 \mathrm{~min}$. As shown in photographs of Figure S18, the non-irradiated 4FCOC showed the delamination of dielectric films due to the soluble solution for COC. By contrast, the crosslinking of 4FCOC inhibit the dissolution of dielectric materials and induce the APTES treatment as shown in contact angle results (Figure S18b). Also, the SAM surface treatment is regarded as the sub nanolength as 2 nm scales. ${ }^{3-5}$ Therefore, The capacitance values of the bilayer structured thin film can be obtained by using the equations to calculate the capacitance values when connecting these points and capacitors in series:

$$
C_{\text {layer }}=\frac{k \varepsilon_{0}}{t}, C_{\text {tot }}=\frac{C_{\text {Polym }} C_{A P T E S}}{C_{\text {polym }}+C_{A P T E S}}
$$

, where $C_{x}(x=$ polym, APTES, tot $)$ is capacitance for each layers, $k$ is dielectric constant, $\varepsilon_{0}$ is vacuum permitivity. According to the previous work ${ }^{6}$, APTES showed the dielectric constant value as 3 5. Cosidering this, the $C_{\text {APTES }}$ exhibits a much larger value than $C_{\text {polym }}$ due to its much thinner thickness ( $300 \mathrm{~nm}$ for Polymer and $\sim 2 \mathrm{~nm}$ for APTES), showing $C_{\text {APTES }}>>>$ $C_{\text {polym }}$. As a results, the relationship rewrites as $C_{\text {Tot }}=C_{\text {polym }} C_{A P T E S} / C_{A P T E S}$. It is difficult to assume that the abrupt change in cap value is caused by APTES through this series of processes. Indeed, the capacitance values shows similar values as pristine $4 \mathrm{FCOC}$ with $6.92 \mathrm{nF} / \mathrm{cm}^{2}$ and APTES treated 4FCOC as $6.74 \mathrm{nF} / \mathrm{cm}^{2}$. Besides, the dielectric constant values were also similar as 2.34 and 2.26 for 4FCOC and APTES treated 4FCOC, respectively. 

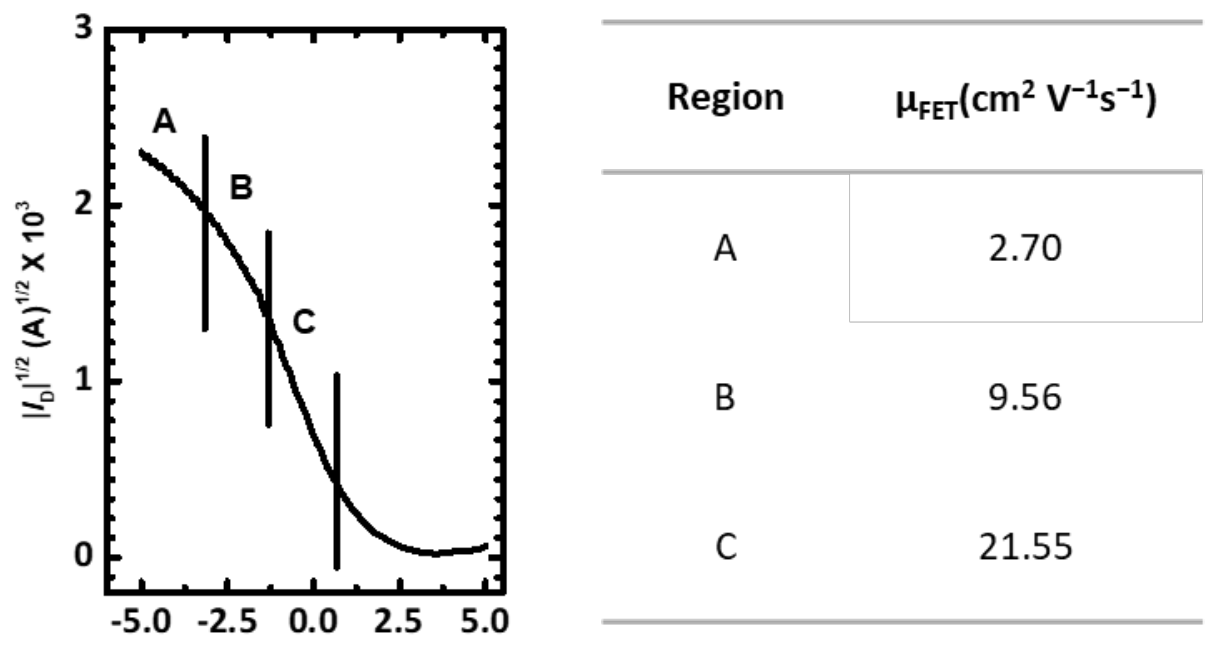

Figure S20. (left) Transfer characteristics in the saturation regime of the OFETs with SWCNT and 4FCOC dielectrics. Each curve was divided into different $V_{\mathrm{G}}$ sweep sections A, B, and C. (right) Summary of the mobility values calculated from each section.

(a)

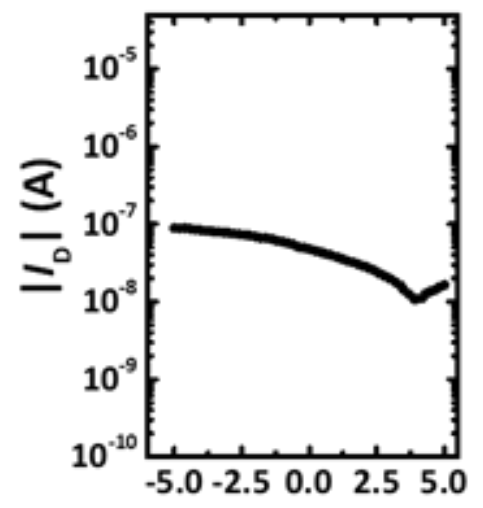

(b)

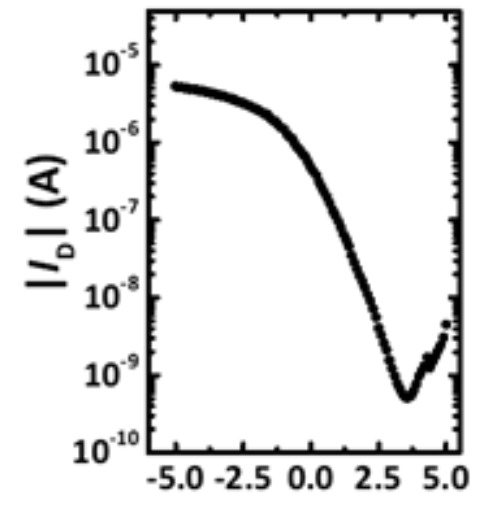

Figure S21, Transfer characteristics of CNT FETs on 4FCOC (a) without or (b) with APTES treatment. 


\section{Supporting References}

(S1) Chua, L.-L.; Ho, P. K.-H.; Friend, R. H.; Manufacture of a polymer device, WO 2004100282 A2, 2004.

(S2) Vig. J. R.; UV/ozone Cleaning of Surfaces, J. Vac. Sci. Technol. A, 1985, 3, 1027-1034.

(S3) Seitz, O.; Fernandes, P. G.; Tian, R.; Karnik, N.; Wen, H.-C.; Stiegler, H.; Chapman, R. A.; Vogela, E. M.; Chabal, Y. J.; Control and Stability of Self-Assembled Monolayers under Biosensing Conditions, J. Mater. Chem., 2011, 21, 4384-4392.

(S4) Casalini, S.; Bortolotti, C. A.; Leonardi, F. Biscarini, F.; Self-Assembled Monolayers in Organic Electronics, Chem. Soc. Rev., 2017, 46, 40-71.

(S5) Vandenberg, E. T.; Bertilsson, L.; Liedberg, B.; Uvdal, K.; Erlandsson, R.; Elwing, H.; Lundström, I.; Structure of 3-Aminopropyl Triethoxy Silane on Silicon Oxide, J. Colloid Interf. Sci. 1991, 147, 103-118.

(S6) Marmisollé, W. A.; Capdevila, D. A.; Llave, E.; Williams, F. J.; Murgida, D. H.; SelfAssembled Monolayers of NH2-Terminated Thiolates: Order, pKa, and Specific Adsorption, Langmuir, 2013, 29, 5351-5359. 